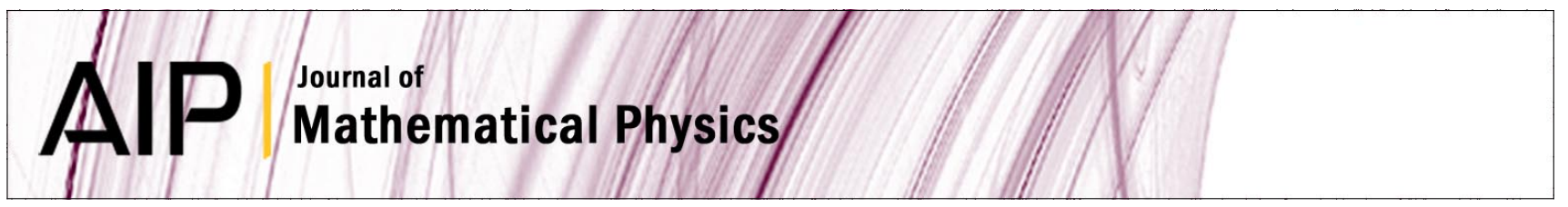

\title{
Lower bounds on blow up solutions of the three-dimensional Navier-Stokes equations in homogeneous Sobolev spaces
}

James C. Robinson, Witold Sadowski, and Ricardo P. Silva

Citation: J. Math. Phys. 53, 115618 (2012); doi: 10.1063/1.4762841

View online: http://dx.doi.org/10.1063/1.4762841

View Table of Contents: http://jmp.aip.org/resource/1/JMAPAQ/v53/i11

Published by the AIP Publishing LLC.

Additional information on J. Math. Phys.

Journal Homepage: http://jmp.aip.org/

Journal Information: http://jmp.aip.org/about/about_the_journal

Top downloads: http://jmp.aip.org/features/most_downloaded

Information for Authors: http://jmp.aip.org/authors

\section{ADVERTISEMENT}

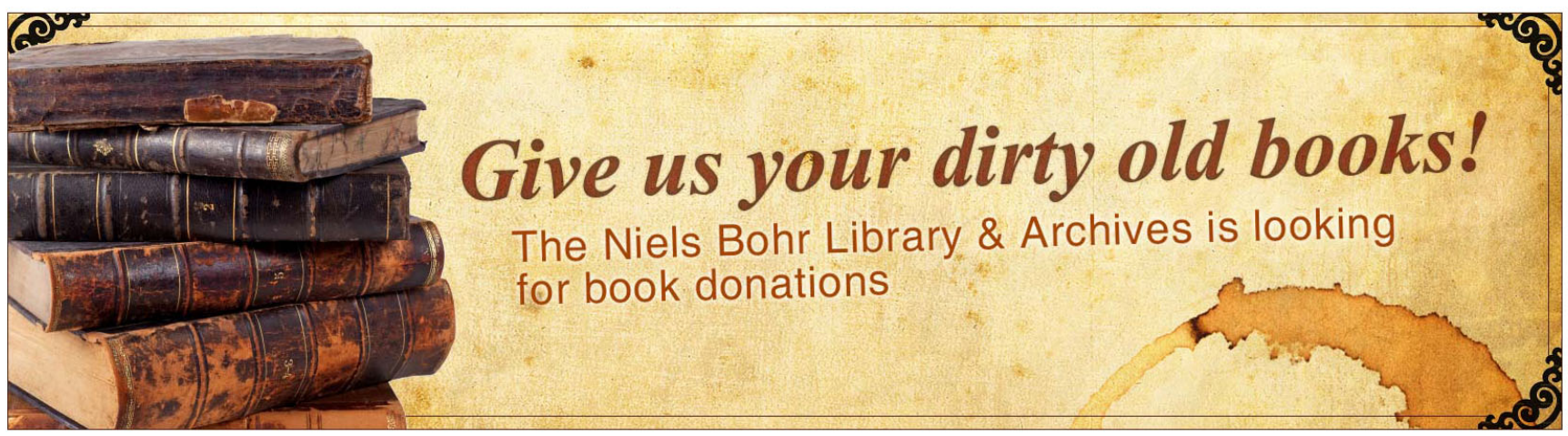




\title{
Lower bounds on blow up solutions of the three-dimensional Navier-Stokes equations in homogeneous Sobolev spaces
}

\author{
James C. Robinson, ${ }^{1}$ Witold Sadowski, ${ }^{2}$ and Ricardo P. Silva ${ }^{3}$ \\ ${ }^{1}$ Mathematics Institute, University of Warwick, Coventry CV4 7AL, United Kingdom \\ ${ }^{2}$ Faculty of Mathematics, Informatics and Mechanics, Warsaw University, Banacha 2, Poland \\ ${ }^{3}$ Instituto de Geociências e Ciências Exatas, UNESP - Univ Estadual Paulista, Departamento \\ de Matemática, 13506-900, Rio Claro SP, Brazil
}

(Received 30 April 2012; accepted 8 October 2012; published online 13 November 2012)

Suppose that $u(t)$ is a solution of the three-dimensional Navier-Stokes equations, either on the whole space or with periodic boundary conditions, that has a singularity at time $T$. In this paper we show that the norm of $u(T-t)$ in the homogeneous Sobolev space $\dot{H}^{s}$ must be bounded below by $c_{s} t^{-(2 s-1) / 4}$ for $1 / 2<s<5 / 2$ $(s \neq 3 / 2)$, where $c_{s}$ is an absolute constant depending only on $s$; and by $c_{s}\left\|u_{0}\right\|_{L^{2}}^{(5-2 s) / 5} t^{-2 s / 5}$ for $s>5 / 2$. (The result for $1 / 2<s<3 / 2$ follows from wellknown lower bounds on blowup in $L^{p}$ spaces.) We show in particular that the local existence time in $\dot{H}^{s}\left(\mathbb{R}^{3}\right)$ depends only on the $\dot{H}^{s}$-norm for $1 / 2<s<5 / 2, s \neq 3 / 2$. (c) 2012 American Institute of Physics. [http://dx.doi.org/10.1063/1.4762841]

To Peter Constantin, on the occasion of his 60th birthday.

\section{INTRODUCTION}

This paper concerns local existence times and lower bounds on putative blow-up solutions for the three-dimensional incompressible Navier-Stokes equations

$$
\frac{\partial u}{\partial t}-\Delta u+(u \cdot \nabla) u+\nabla p=0, \quad \nabla \cdot u=0,
$$

posed either on $\mathbb{R}^{3}$ or on a periodic cube $Q=[0,2 \pi]^{3}$ with $\int_{Q} u=0$.

In his seminal paper on the three-dimensional Navier-Stokes equations (on the whole space $\mathbb{R}^{3}$ ), Leray (1934) showed (p. 224) that if a smooth solution loses regularity at time $T$ then necessarily the $\dot{H}^{1}$-norm must blow up with the lower bound

$$
\|u(T-t)\|_{\dot{H}^{1}\left(\mathbb{R}^{3}\right)} \geq c_{1}(T-t)^{-1 / 4} .
$$

He also gave (without proof, p. 227) lower bounds for such "blowing up" solutions in the Lebesgue spaces, namely

$$
\|u(T-t)\|_{L^{p}\left(\mathbb{R}^{3}\right)} \geq \kappa_{p} t^{-(p-3) / 2 p} \quad 3<p<\infty ;
$$

proofs of this lower bound have been given by Giga (1986) using the semigroup approach and by Robinson and Sadowski (2012) using elementary energy estimates. If one combines this $L^{p}$ blowup with the Sobolev embedding $\dot{H}^{s}\left(\mathbb{R}^{3}\right) \subset L^{6 /(3-2 s)}\left(\mathbb{R}^{3}\right)$ then one can deduce that

$$
\|u(T-t)\|_{\dot{H}^{s}\left(\mathbb{R}^{3}\right)} \geq c_{s} t^{-(2 s-1) / 4},
$$

for any $1 / 2<s<3 / 2$.

Note that in all these cases the solutions are smooth (belong to $\dot{H}^{s}$ for every $s$ ) for $t>0$ and remain so until the blow-up time; in particular all the formal manipulations we will carry out in what follows are rigorous. Furthermore the blowup time coincides in all these spaces; this follows from 
the interpolations

$$
\|u\|_{\dot{H}^{1 / 2}} \leq c\|u\|_{L^{2}}^{(2 s-1) / 2 s}\|u\|_{\dot{H}^{s}}^{1 / 2 s}, \quad s>1 / 2,
$$

and

$$
\|u\|_{L^{3}} \leq c\|u\|_{L^{2}}^{2(p-3) / 3(p-2)}\|u\|_{L^{p}}^{p / 3(p-2)}, \quad 3<p<\infty,
$$

since any solution that remains bounded in $\dot{H}^{1 / 2}$ or $L^{3}$ must be smooth (see Seregin (2011) for $\dot{H}^{1 / 2}$, and Seregin (2012) for $\left.L^{3}\right)$.

Both (1.1) and (1.2) are examples of "optimal" blowup rates, having the "correct" rate of blowup with respect to the scaling of the various norms under the transformation $u(x, t) \mapsto \lambda u(\lambda x$, $\lambda^{2} t$ ) which maps any solution of the Navier-Stokes equations to another solution; under rescaling $\|u\|_{H^{s}} \sim \lambda^{s-(1 / 2)}$ and $t \sim \lambda^{-2}$. In Sec. IV we extend the lower bound in (1.2) to cover $3 / 2<s$ $<5 / 2$, and give an argument which shows that this rate of blowup also occurs in the case of periodic boundary conditions.

Recently, Benameur (2010) investigated lower bounds on blowup solutions in $\dot{H}^{s}\left(\mathbb{R}^{3}\right)$ for $s>5 / 2$, and obtained the result

$$
\|u(T-t)\|_{\dot{H}^{s}\left(\mathbb{R}^{3}\right)} \geq \gamma_{s}\|u(T-t)\|_{L^{2}\left(\mathbb{R}^{3}\right)}^{(3-2 s) / 3} t^{-s / 3} .
$$

In this paper, we obtain an improved lower bound for this range of $s$, namely

$$
\|u(T-t)\|_{\dot{H}^{s}\left(\mathbb{R}^{3}\right)} \geq c_{s}\left\|u_{0}\right\|_{L^{2}\left(\mathbb{R}^{3}\right)}^{(5-2 s) / 5} t^{-2 s / 5} .
$$

Note that these both respect the rescaling of solutions, but the new lower bound is asymptotically greater than the first.

We show in Sec. II how these results can be obtained on the whole space by simple scaling arguments: (1.2) follows if the local existence time depends only on the norm in $\dot{H}^{s}\left(\mathbb{R}^{3}\right)$, while (1.4) can be deduced from the fact the local existence time is at least $c_{s}\left\|u_{0}\right\|_{H^{s}\left(\mathbb{R}^{3}\right)}^{-1}$ (i.e. involves the full $H^{s}$ norm). Indeed, lower bounds for blowup and for local existence times are in some sense dual to each other: suppose that given an initial condition $u_{0}$ in a space $X$ there exists a time $T\left(\left\|u_{0}\right\|_{X}\right)$ such that the solution exists and remains in $X$ on the time interval $[0, T]$. Whatever the functional form of $T$, this gives a necessary lower bound on the $X$-norm of any solution that blows up at time $T^{*}$, given implicitly by the relation

$$
T\left(\left\|u\left(T^{*}-t\right)\right\|_{X}\right)<t \quad \text { for every } t \in\left(0, T^{*}\right] .
$$

For particular functions $T$ such as power laws this is simple; clearly

$$
T(a)=c a^{-\alpha} \quad \Rightarrow \quad\left\|u\left(T^{*}-t\right)\right\|_{X} \geq c^{\prime} t^{-1 / \alpha} .
$$

Similarly if there is a function $\varphi$ such that any solution that blows up at time $T^{*}$ must satisfy

$$
\left\|u\left(T^{*}-t\right)\right\|_{X} \geq \varphi(t)
$$

it follows that if $\left\|u_{0}\right\|<\varphi(T)$ then the solution exists on $[0, T]$.

In Secs. IV and VI we give direct proofs of the above lower bounds on blowup solutions, which are thus also valid in the periodic case, by proving upper bounds on the nonlinear term

$$
\left|(B(u, u), u)_{\dot{H}^{s}}\right| \leq \begin{cases}c_{s}\|u\|_{s}\|u\|_{s+1}\|\hat{u}\|_{L^{1}} & s \geq 0 \\ c_{s}\|u\|_{s}^{2}\|\widehat{D u}\|_{L^{1}} & s \geq 1\end{cases}
$$

and the interpolation result

$$
\|\hat{u}\|_{L^{1}} \leq\|u\|_{s}^{s-1 / 2}\|u\|_{s+1}^{3 / 2-s} \text { for } 1 / 2<s<3 / 2 .
$$

Here, and throughout the paper, we use $\|u\|_{s}$ to denote the norm of $u$ in $\dot{H}^{s}$ (for formal definitions see Sec. III). These inequalities, inspired by Lemma 10.4 in Constantin and Foias (1988), are proved in Sec. III, which is really the heart of the paper.

We denote throughout by $c_{s}$ any absolute constant that depends only on $s$; this may change from line to line. 


\section{BLOW UP AND SCALING FOR SOLUTIONS ON THE WHOLE SPACE}

We now make some elementary observations that are based on the fact that solutions remain solutions under the scaling

$$
u(x, t) \mapsto u_{\lambda}(x, t):=\lambda u\left(\lambda x, \lambda^{2} t\right) .
$$

In particular, if $u$ denotes the solution with initial condition $u_{0}$ and $u_{\lambda}$ denotes the solution corresponding to the initial condition $u_{0, \lambda}=\lambda u(\lambda x)$ then

$$
u_{\lambda} \text { is regular on }\left(0, T_{\lambda}\right) \Leftrightarrow u \text { is regular on }\left(0, \lambda^{2} T_{\lambda}\right) .
$$

We say that a space $X$ scales like $\lambda^{\alpha}$ if $\left\|u_{0, \lambda}\right\|=\lambda^{\alpha}\left\|u_{0}\right\|_{X}$ for all $u_{0} \in X$, and that $X$ is scaling-invariant if $\left\|u_{0, \lambda}\right\|_{X}=\left\|u_{0}\right\|_{X}$.

An immediate observation is that if one could find a local existence time that depended only on the norm in a scale-invariant space $X$ (such as $\dot{H}^{1 / 2}$ or $L^{3}$ ) then this would imply global existence for all solutions; for any $\lambda>0$ the initial condition $\left(u_{0}\right)_{\lambda}$ has the same $X$-norm, and one can choose $\lambda$ such that $\lambda^{2}\left\|u_{0}\right\|_{X}$ is as large as we please. All the known local existence results in such critical spaces depend on the structure of $u_{0}$ itself and not only on its norm in the appropriate space $\left(L^{3}\right.$ in Giga, 1986; Robinson and Sadowski, 2012; $\dot{H}^{1 / 2}$ in Fujita and Kato, 1964; Chemin et al., 2006; Marín-Rubio et al., 2012; $\mathrm{BMO}^{-1}$ in Koch and Tataru, 2001; for a general treatment see Cannone, 1995, 2003; Lemarié-Rieusset, 2002).

Now suppose that the Navier-Stokes equations on the whole space are not regular and that a certain $u_{0} \in X$, where $X$ scales like $\lambda^{\alpha}$ with $\alpha \neq 0$, gives rise to a solution $u$ that blows up at time $T$. Then for any $\lambda>0$ the initial condition $u_{0, \lambda}$ gives rise to the solution $u_{\lambda}$ that also blows up in a finite time. Since $\left\|u_{0, \lambda}\right\|_{X}=\lambda^{\alpha}\left\|u_{0}\right\|_{X}$ it follows that for each $a>0$ there is a solution $v$ with an initial condition $\left\|v_{0}\right\|_{X}=a$ that blows up in a finite time. Therefore on the whole space no smallness of initial data in $X$ could prevent blowup (if it occurs for any initial condition).

Let us denote by $T_{X}\left(u_{0}\right)$ the maximal interval of existence in $X$ of the solution starting at $u_{0} \in X$. Most local existence results give lower bounds for $T_{X}\left(u_{0}\right)$; in the following two lemmas we give simple proofs of the blowup rates in the homogeneous Sobolev spaces on the whole $\mathbb{R}^{3}$, using only assumptions about the dependence of these lower bounds on various norms and rescaling arguments.

Lemma 2.1. Let $X$ be a space that scales like $\lambda^{\alpha}, \alpha>0$. If

$$
T_{X}\left(u_{0}\right) \geq \tau\left(\left\|u_{0}\right\|_{X}\right)>0,
$$

for some function $\tau:[0, \infty) \rightarrow(0, \infty)$ then for some $c_{X}>0$

$$
T_{X}\left(u_{0}\right) \geq c_{X}\left\|u_{0}\right\|_{X}^{-2 / \alpha},
$$

and so in particular if $u$ blows up at time $T$ then

$$
\|u(T-t)\|_{X} \geq c_{X}^{\prime} t^{-\alpha / 2} .
$$

Furthermore this rate is optimal: if any solution that blows up must satisfy

$$
\|u(T-t)\|_{X} \geq c t^{-\gamma}, \quad \text { for some } \quad \gamma>\alpha / 2,
$$

then in fact there can be no blowup.

Proof. We give two proofs of (2.1), one very short and one that shows that the rate we obtain cannot be improved by scaling arguments. First, given any non-zero $u_{0} \in X$ consider the initial condition $u_{0, \lambda}$ with $\lambda=\left\|u_{0}\right\|_{X}^{-1 / \alpha}$. Since $\left\|u_{0, \lambda}\right\|_{X}=1$ it follows that

$$
T_{X}\left(u_{0}\right) \geq \tau(1)\left\|u_{0}\right\|_{X}^{-2 / \alpha} .
$$

It is not immediately clear from this argument that the result is optimal. Instead let us consider

$$
\hat{T}_{X}(a):=\sup _{\lambda>0} \lambda^{2} \tau\left(\lambda^{\alpha} a\right) .
$$


By construction $\hat{T}_{X}$ is invariant under this rescaling:

$$
\begin{aligned}
\mu^{2} \hat{T}_{X}\left(\mu^{\alpha} a\right) & =\sup _{\lambda>0}(\mu \lambda)^{2} \tau\left((\mu \lambda)^{\alpha} a\right) \\
& =\sup _{(\lambda \mu)>0}(\mu \lambda)^{2} \tau\left((\mu \lambda)^{\alpha} a\right) \\
& =\hat{T}_{X}(a),
\end{aligned}
$$

it follows that $\hat{T}_{X}(a)=c a^{-2 / \alpha}$.

To show that this rate is indeed optimal, suppose that $\|u(T-t)\|_{X} \geq c t^{-\gamma}$ for some $\gamma>\alpha / 2$, for any solution that blows up. Then the local existence time starting at some $u_{0}$ is at least $c\left\|u_{0}\right\|_{X}^{-1 / \gamma}$. Now, using the rescaling argument above, the local existence time is at least

$$
\sup _{\lambda>0} c \lambda^{2}\left[\lambda^{\alpha}\left\|u_{0}\right\|_{X}\right]^{-1 / \gamma}=\sup _{\lambda>0} c \lambda^{2-\alpha / \gamma}\left\|u_{0}\right\|_{X}^{-1 / \gamma}=\infty,
$$

so there is no blowup.

Since $\dot{H}^{s}\left(\mathbb{R}^{3}\right)$ scales like $\lambda^{s-1 / 2}$ it follows that if the local existence time depends only on the norm in $\dot{H}^{s}\left(\mathbb{R}^{3}\right)$ (this is the case for $1 / 2<s<5 / 2, s \neq 3 / 2$, see Sec. IV) then

$$
\|u(T-t)\|_{\dot{H}^{s}\left(\mathbb{R}^{3}\right)} \geq c t^{-(2 s-1) / 4},
$$

which we refer to as the "optimal rate" for blowup in $\dot{H}^{s}$ spaces.

For $s>5 / 2$ we can show that the existence time is bounded below by

$$
c_{s}^{\prime}\left\|u_{0}\right\|_{H^{s}\left(\mathbb{R}^{3}\right)}^{-1} \geq c_{s}\left(\|u\|_{L^{2}\left(\mathbb{R}^{3}\right)}^{2}+\|u\|_{H^{s}\left(\mathbb{R}^{3}\right)}^{2}\right)^{-1 / 2}
$$

(cf. Majda and Bertozzi (2002) and see Sec. VI). This also has an immediate consequence for the blowup rate of solutions in $\dot{H}^{s}\left(\mathbb{R}^{3}\right)$.

Lemma 2.2. Suppose that the local existence time in $H^{s}\left(\mathbb{R}^{3}\right), s>5 / 2$, depends on the norm in $H^{s}\left(\mathbb{R}^{3}\right)$, with

$$
T_{s}\left(u_{0}\right) \geq \frac{c_{s}^{\prime}}{\left\|u_{0}\right\|_{H^{s}\left(\mathbb{R}^{3}\right)}} .
$$

Then

$$
T_{s}\left(u_{0}\right) \geq c_{s}\left\|u_{0}\right\|_{L^{2}\left(\mathbb{R}^{3}\right)}^{(5-2 s) / 2 s}\left\|u_{0}\right\|_{\dot{H}^{s}\left(\mathbb{R}^{3}\right)}^{-5 / 2 s}
$$

and if the solution blows up at time T then

$$
\|u(T-t)\|_{\dot{H}^{s}\left(\mathbb{R}^{3}\right)} \geq c_{s}\|u(T-t)\|_{L^{2}\left(\mathbb{R}^{3}\right)}^{(5-2) / 5} t^{-2 s / 5} .
$$

As remarked by Benameur, we note that if $s>5 / 2$ then the $L^{2}$ factor is bounded below so does not "detract" from the rate of blowup.

Proof. Let $u_{0}$ be such that $\left\|u_{0}\right\|_{L^{2}}^{2}=a$ and $\left\|u_{0}\right\|_{\dot{H}^{s}}^{2}=b$. Then $\left\|u_{0, \lambda}\right\|_{L^{2}}^{2}=\lambda^{-1} a$ and $\left\|u_{0, \lambda}\right\|_{\dot{H}^{s}}^{2}$ $=\lambda^{2 s-1} b$. The rescaled solution $u_{\lambda}$ is regular on $\left(0, T_{\lambda}\right)$, where $T_{\lambda} \geq C_{s}\left(\lambda^{-1} a+\lambda^{2 s-1} b\right)^{-1 / 2}$. Therefore $u$ is regular on $\left(0, \lambda^{2} T_{\lambda}\right)$. The maximum value of $f(\lambda)=\lambda^{2} T_{\lambda}$ occurs at $\lambda_{0}$, where $\lambda_{0}^{2 s}=5 a /(2 s-5) b$, and is

$$
f\left(\lambda_{0}\right)=c_{s}\left\|u_{0}\right\|_{L^{2}}^{(5-2 s) / 2 s}\left\|u_{0}\right\|_{\dot{H}^{s}}^{-5 / 2 s},
$$

where $c_{s}$ depends only on $s$. The lower bound in (2.3) follows immediately. (In fact, modulo the constant, it suffices to take $\lambda$ to equalise the two terms in the denominator, i.e., $\lambda^{2 s}=a / b$.)

A similar argument to that used in the proof of Lemma 2.2 can in fact be used to obtain Benameur's bound (1.3) very quickly and for a larger range of $s$, namely $s>3 / 2$ rather than only 
$s>5 / 2$. Indeed, Benameur shows (and we will prove the same result in Lemma 5.1 in the periodic setting) that the local existence time can be bounded below by

$$
c\|\hat{u}\|_{L^{1}}^{-2} .
$$

(This result is well known and relatively easy to obtain using the variation of constants formula for solutions.) Since straightforward computations show that $\|\hat{u}\|_{L^{1}} \leq c_{s}^{\prime}\|u\|_{H^{s}}$ for any $s>3 / 2$ (see (3.4) in Sec. III) we get, for any $s>3 / 2$,

$$
T \geq \frac{c}{\|\hat{u}\|_{L^{1}}^{2}} \geq \frac{C}{\|u\|_{H^{s}}^{2}}=\frac{C}{a+b},
$$

where as above we write $a=\|u\|_{L^{2}}^{2}$ and $b=\|u\|_{\dot{H}^{s}}^{2}$. Now we can apply the scaling method of the above proof. The rescaled solution is regular on

$$
\lambda^{2} T_{\lambda}=\frac{\lambda^{3}}{a+\lambda^{2 s} b},
$$

and if we set $\lambda^{2 s}=a / b$ then the bound in (1.3),

$$
\|u(T-t)\|_{\dot{H}^{s}} \geq c_{s}\|u(T-t)\|_{L^{2}}^{(3-2 s) / 3} t^{-s / 3}
$$

follows immediately; but note that using this argument we can obtain this inequality for all $s>3 / 2$ and not only $s>5 / 2$. (The same result is obtained, with a slightly better constant, if one maximises the right-hand side of (2.5).)

Note, however, that while the estimate in (2.6) is valid for all $s>3 / 2$, it is far from being optimal in the range $s>5 / 2$, where the estimate (2.3) is stronger. (For $s \rightarrow 3 / 2$ the estimate (2.6) gives in the limit the "optimal rate" $\sim t^{-1 / 2}$, but for $s=5 / 2$ we have $\sim t^{-5 / 6}$ which is much worse than the optimal rate equal to the limit of (2.3) when $s \rightarrow 5 / 2$.)

\section{INEQUALITIES FOR THE NONLINEAR TERM}

Since scaling arguments are not available in the periodic case our analysis will rely on careful energy estimates. Central to these will be the following bounds on the nonlinear term, inspired by and similar to those in Lemma 10.4 in Constantin and Foias (1988). We prove these estimates in the periodic case, but they also hold in the full space, replacing Fourier series by Fourier integrals. When the results are true in both cases we will omit the domain from the space in the norm, see Lemma 3.1, for example.

Let $Q=[0,2 \pi]^{3}$. We write $\dot{\mathbb{Z}}^{3}=\mathbb{Z}^{3} \backslash\{0,0,0\}$, let $\dot{H}^{s}(Q)$ be the subspace of the Sobolev space $H^{s}$ consisting of divergence-free, zero-average, periodic real functions,

$$
\dot{H}^{s}(Q)=\left\{u=\sum_{k \in \dot{\mathbb{Z}}^{3}} \hat{u}_{k} \mathrm{e}^{\mathrm{i} k \cdot x}: \hat{u}_{k}=\overline{\hat{u}_{-k}}, \sum|k|^{2 s}\left|\hat{u}_{k}\right|^{2}<\infty, k \cdot \hat{u}_{k}=0\right\},
$$

and equip $\dot{H}^{s}(Q)$ with the norm

$$
\|u\|_{s}^{2}=\sum|k|^{2 s}\left|\hat{u}_{k}\right|^{2} .
$$

On the whole space the corresponding definition of the $\dot{H}^{s}$-norm is

$$
\|u\|_{\dot{H}^{s}\left(\mathbb{R}^{3}\right)}^{2}:=\int_{\mathbb{R}^{3}}|k|^{2 s}|\hat{u}(k)|^{2} \mathrm{~d} k,
$$

where

$$
\hat{u}(k)=\int_{\mathbb{R}^{3}} \mathrm{e}^{-\mathrm{i} k \cdot x} u(x) \mathrm{d} x .
$$

Let $\Pi$ be the orthogonal projection in $\left[L^{2}(Q)\right]^{3}$ onto divergence-free vector fields, and denote by $A$ the Stokes operator on $Q$, that is,

$$
A=-\Pi \Delta .
$$


In the periodic case $A u=-\Delta \Pi u$, so $A u=-\Delta u$ for $u \in \dot{H}^{s}(Q)$. We make continual use of the equivalence of the norms $\|u\|_{s}=\|u\|_{\dot{H}^{s}}$ and $\left\|A^{s / 2} u\right\|$ for $u \in \dot{H}^{s}(Q)=D\left(A^{s / 2}\right)$.

We denote by $B(u, u)$ the bilinear form defined by

$$
B(u, u)=\Pi[(u \cdot \nabla) u] .
$$

The Navier-Stokes equations can then be written as

$$
u_{t}+A u+B(u, u)=0 .
$$

For further details see. Constantin and Foias (1988)

In our estimates we will also use the $L^{1}$-type norms of the Fourier transform,

$$
\|u\|_{F^{s}(Q)}:=\sum_{k \in \dot{\mathbb{Z}}^{3}}|k|^{s}\left|\hat{u}_{k}\right|
$$

and

$$
\|u\|_{F^{s}\left(\mathbb{R}^{3}\right)}=\int_{\mathbb{R}^{3}}|k|^{s}|\hat{u}(k)| \mathrm{d} k .
$$

We denote by $F^{s}$ the space of all those $u$ for which $\|u\|_{F^{s}}$ is finite and write $\|u\|_{F}$ for $\|u\|_{F^{0}}$. Similar norms have been used in the context of generalised Gevrey-type spaces by Oliver and Titi (2001).

Note that $F^{0}(Q) \subset L^{\infty}(Q)$, since

$$
\|u\|_{L^{\infty}} \leq \sum\left|\hat{u}_{k}\right|=\|u\|_{F},
$$

and similarly $F^{0}\left(\mathbb{R}^{3}\right) \subset L^{\infty}\left(\mathbb{R}^{3}\right)$. In the periodic case

$$
\begin{aligned}
\|u\|_{F^{s}(Q)} & =\sum_{k}|k|^{s}\left|\hat{u}_{k}\right| \\
& \leq\left(\sum_{k}|k|^{-2 \alpha}\right)^{1 / 2}\left(\sum_{k}|k|^{2(s+\alpha)}\left|\hat{u}_{k}\right|^{2}\right)^{1 / 2} \\
& =c_{\alpha}\|u\|_{s+\alpha},
\end{aligned}
$$

for any $\alpha>3 / 2$, so in particular $\dot{H}^{s+\alpha}(Q) \subset F^{s}(Q)$ for $\alpha>3 / 2$. On the whole space (cf. (24) in Oliver and Titi (2000))

$$
\begin{aligned}
\|u\|_{F^{s}\left(\mathbb{R}^{3}\right)} & =\int_{\mathbb{R}^{3}}|k|^{s}|\hat{u}(k)| \\
& \leq\left(\int_{\mathbb{R}^{3}}\left(1+|k|^{2}\right)^{-\alpha} \mathrm{d} k\right)^{1 / 2}\left(\int_{\mathbb{R}^{3}}\left(1+|k|^{2}\right)^{s+\alpha}|\hat{u}(k)|^{2} \mathrm{~d} k\right)^{1 / 2} \\
& \leq c_{\alpha}\|u\|_{H^{s+\alpha}\left(\mathbb{R}^{3}\right)}
\end{aligned}
$$

we need the full Sobolev norm to bound the $F^{s}$ norm (but see also (3.10)).

Lemma 3.1. For any $s \geq 0$

$$
\left\|A^{s / 2} B(u, v)\right\|_{L^{2}} \leq c\left\{\|u\|_{F}\|v\|_{s+1}+\|v\|_{F}\|u\|_{s+1}\right\} \quad \text { for all } \quad u, v \in \dot{H}^{s+1} \cap F^{0}
$$

and for any $s \geq 1$

$$
\left|\left(A^{s} B(u, u), u\right)_{L^{2}}\right| \leq c\|u\|_{F^{1}}\|u\|_{s}^{2} \text { for all } u \in \dot{H}^{s} \cap F^{1} .
$$

These inequalities are valid for periodic boundary conditions and on the whole of $\mathbb{R}^{3}$.

In what follows we will primarily make use of (3.5) in the form

$$
\left\|A^{s / 2} B(u, u)\right\|_{L^{2}} \leq c\|u\|_{F}\|u\|_{s+1} .
$$


Proof. To prove both inequalities we use the Fourier expansion

$$
u=\sum_{k \in \dot{\mathbb{Z}}^{3}} \hat{u}_{k} \mathrm{e}^{\mathrm{i} k \cdot x}
$$

proofs on the whole space follow exactly the same steps, replacing the Fourier series by a Fourier integral and sums over $\dot{\mathbb{Z}}^{3}$ by integrals over $\mathbb{R}^{3}$.

For the first inequality (proved in the whole space case for $u=v$ by Benameur (2010)) we estimate $\left(A^{s / 2} B(u, v), w\right)$ for $w \in L^{2}$ (in fact first we take $w \in D\left(A^{s / 2}\right)$, and then pass to the limit in our final inequality). Since $v$ is divergence free we have $\hat{v}_{k-j} \cdot(k-j)=0$ and so

$$
\begin{aligned}
\left(A^{s / 2} B(u, v), w\right) & =\left(B(u, v), A^{s / 2} w\right) \\
& =\mathrm{i} \sum_{j, k}\left(j \cdot \hat{v}_{k-j}\right)\left(\hat{u}_{j} \cdot \hat{w}_{k}\right)|k|^{s} \\
& =\mathrm{i} \sum_{j, k}\left(k \cdot \hat{v}_{k-j}\right)\left(\hat{u}_{j} \cdot \hat{w}_{k}\right)|k|^{s},
\end{aligned}
$$

hence, since $\hat{w}_{-k}=\overline{\hat{w}}_{k}$,

$$
\begin{aligned}
\left|\left(A^{s / 2} B(u, v), w\right)\right| & \leq \sum_{j, k}\left|\hat{v}_{k-j}\left\|\hat{u}_{j}\right\| \hat{w}_{k} \| k\right|^{s+1} \\
& \leq 2^{s+1} \sum_{j, k}\left|\hat{v}_{k-j}\left\|\hat{u}_{j}\right\| \hat{w}_{k}\right|\left(|k-j|^{s+1}+|j|^{s+1}\right) \\
& =2^{s+1} \sum_{i, j}\left|\hat{v}_{i}\left\|\hat{u}_{j}\right\| \hat{w}_{i+j}\right|\left(|i|^{s+1}+|j|^{s+1}\right) \\
& =2^{s+1} \sum_{i, j}|i|^{s+1}\left|\hat{v}_{i}\left\|\hat{u}_{j}\right\| \hat{w}_{i+j}\right|+\left|\hat { v } _ { i } \left\|\left.j\right|^{s+1}\left|\hat{u}_{j} \| \hat{w}_{i+j}\right|\right.\right. \\
& \leq 2^{s+1} \sum_{j}\left(\left|\hat{u}_{j}\right| \sum_{i}|i|^{s+1}\left|\hat{v}_{i} \| \hat{w}_{i+j}\right|\right)+2^{s+1} \sum_{i}\left(\left|\hat{v}_{i}\right| \sum_{j}|j|^{s+1}\left|\hat{u}_{j} \| \hat{w}_{i+j}\right|\right) \\
& \leq 2^{s+1}\left(\sum_{j}\left|\hat{u}_{j}\right|\right)\|v\|_{s+1}\|w\|+2^{s+1}\left(\sum_{i}\left|\hat{v}_{i}\right|\right)\|u\|_{s+1}\|w\| \\
& \leq c_{s}\left(\|u\|_{F}\|v\|_{s+1}+\|v\|_{F}\|u\|_{s+1}\right)\|w\| .
\end{aligned}
$$

For the second inequality we note, following Constantin and Foias, that

$$
\left(B\left(u, A^{s / 2} u\right), A^{s / 2} u\right)=0,
$$

and so

$$
\left(A^{s} B(u, u), u\right)=\left(A^{s / 2} B(u, u)-B\left(u, A^{s / 2} u\right), A^{s / 2} u\right) .
$$

We just need to estimate

$$
\left\|A^{s / 2} B(u, u)-B\left(u, A^{s / 2} u\right)\right\|_{L^{2}}
$$

which we do by considering

$$
I:=\left(A^{s / 2} B(u, u)-B\left(u, A^{s / 2} u\right), w\right),
$$

for $\|w\|_{L^{2}}=1$ (again we take $w \in D\left(A^{s / 2}\right)$ and pass to the limit). Now,

$$
\left(A^{s / 2} B(u, u), w\right)=\left(B(u, u), A^{s / 2} w\right)=\mathrm{i} \sum_{j, k}\left(j \cdot \hat{u}_{k-j}\right)\left(\hat{u}_{j} \cdot \hat{w}_{k}\right)|k|^{s}
$$


and

$$
\left(B\left(u, A^{s / 2} u\right), w\right)=\mathrm{i} \sum_{j, k}\left(j \cdot \hat{u}_{k-j}\right)\left(\hat{u}_{j} \cdot \hat{w}_{k}\right)|j|^{s}
$$

Thus

$$
I=\mathrm{i} \sum_{j, k}\left(j \cdot \hat{u}_{k-j}\right)\left(\hat{u}_{j} \cdot \hat{w}_{k}\right)\left(|k|^{s}-|j|^{s}\right),
$$

and so

$$
|I| \leq\left.\sum_{j, k}\left|j\left\|\hat{u}_{k-j}\right\| \hat{u}_{j} \| \hat{w}_{k}\right||| k\right|^{s}-|j|^{s} \mid .
$$

By writing

$$
|k|^{s}-|j|^{s}=\int_{0}^{1} \frac{\mathrm{d}}{\mathrm{d} t}|j+t(k-j)|^{s} \mathrm{~d} t,
$$

it is easy to show that for any $s \geq 1$

$$
\left.|| k\right|^{s}-|j|^{s}\left|\leq s 2^{s-1}\right| k-j \mid\left[|k-j|^{s-1}+|j|^{s-1}\right] .
$$

It follows that

$$
\begin{aligned}
|I| & \leq s 2^{s-1} \sum_{j, k}\left(|j|^{s}|k-j|+|k-j|^{s}|j|\right)\left|\hat{u}_{k-j}\left\|\hat{u}_{j}\right\| \hat{w}_{k}\right| \\
& =s 2^{s-1} \sum_{i, j}\left(|j|^{s}|i|+|i|^{s}|j|\right)\left|\hat{u}_{i}\left\|\hat{u}_{j}\right\| \hat{w}_{i+j}\right| \\
& =s 2^{s} \sum_{i}\left(\left|i\left\|\left.\hat{u}_{i}\left|\sum_{j}\right| j\right|^{s}\left|\hat{u}_{j} \| \hat{w}_{i+j}\right|\right)\right.\right. \\
& \leq c_{s}\left(\sum_{i}\left|i \| \hat{u}_{i}\right|\right)\|u\|_{s}\|w\|
\end{aligned}
$$

where $c_{s}=s 2^{s}$.

Our bounds are similar to those in Lemma 10.4 in Constantin and Foias (1988), but they choose to bound the $F$ norms by Sobolev norms using

$$
\|u\|_{F(Q)} \leq c_{\alpha}\|u\|_{\alpha} \quad \text { for any } \quad \alpha>3 / 2
$$

(see (3.3)), obtaining

$$
\left\|A^{s / 2} B(u, u)\right\|_{L^{2}(Q)} \leq c\|u\|_{s}\|u\|_{s+1} \quad \text { for all } \quad s>3 / 2
$$

and

$$
\left|\left(A^{s} B(u, u), u\right)_{L^{2}(Q)}\right| \leq c\|u\|_{s}^{3} \quad \text { for all } \quad s>5 / 2 .
$$

The advantage of writing the inequalities in the form of Lemma 3.1 is that it becomes clear that the estimates "improve" as $s$ grows.

A stronger version of (3.5), whose proof (Klainerman and Majda, 1981) is only valid for integer values of $s$, is used in the celebrated paper by Beale et al. (1984) that obtains necessary and sufficient conditions for blowup of solutions of the Euler equation, namely

$$
((B(u, u), u))_{H^{s}} \leq c\|D u\|_{L^{\infty}}\|u\|_{H^{s}}^{2}, \quad s \in \mathbb{N}, s \geq 1,
$$


where

$$
((u, v))_{H^{s}}=\sum_{|\alpha| \leq s}\left(\partial^{\alpha} u, \partial^{\alpha} v\right) .
$$

However, note that this involves the full $H^{s}$ norm, rather than the homogeneous norm.

If one wants to use Sobolev norms (which we do), we can improve on (3.8): if we had this bound with $\alpha=3 / 2$ then for $1 / 2<s<3 / 2$ (for example) we could use Sobolev interpolation to obtain

$$
\|u\|_{F} \leq c\|u\|_{s}^{s-1 / 2}\|u\|_{s+1}^{3 / 2-s} .
$$

That such interpolation-type inequalities do in fact hold for the $F^{r}$-norms is the content of the following lemma.

Lemma 3.2. If $0 \leq s_{1}<3 / 2+r<s_{2}$ and $u \in \dot{H}^{s_{1}} \cap \dot{H}^{s_{2}}$ then $u \in F^{r}$ and

$$
\|u\|_{F^{r}} \leq c\|u\|_{s_{1}}^{\left(s_{2}-r-3 / 2\right) /\left(s_{2}-s_{1}\right)}\|u\|_{s_{2}}^{\left(3 / 2+r-s_{1}\right) /\left(s_{2}-s_{1}\right)} \text {. }
$$

Proof. We have

$$
\begin{aligned}
& \sum_{k}|k|^{r}|\hat{u}(k)|= \sum_{|k| \leq K}|k|^{r}|\hat{u}(k)|+\sum_{|k| \geq K}|k|^{r}|\hat{u}(k)| \\
& \leq\left(\sum_{|k| \leq K}|k|^{2\left(r-s_{1}\right)}\right)^{1 / 2}\left(\sum_{|k| \leq K}|k|^{2 s_{1}}|\hat{u}(k)|^{2}\right)^{1 / 2} \\
&+\left(\sum_{|k| \geq K}|k|^{2\left(r-s_{2}\right)}\right)^{1 / 2}\left(\sum_{|k| \geq K}|k|^{2 s_{2}}|\hat{u}(k)|^{2}\right)^{1 / 2} \\
& \leq c K^{\left(3 / 2+r-s_{1}\right)}\|u\|_{s_{1}}+c K^{\left(3 / 2+r-s_{2}\right)}\|u\|_{s_{2}} .
\end{aligned}
$$

Now choose $K=\left(\|u\|_{s_{2}} /\|u\|_{s_{1}}\right)^{1 /\left(s_{2}-s_{1}\right)}$ to obtain (3.10).

We also note the interpolation inequality in the $F^{r}$ spaces, for $s_{1}<r<s_{2}$

$$
\|u\|_{F^{r}} \leq\|u\|_{F^{s_{1}}}^{\left(s_{2}-r\right) /\left(s_{2}-s_{1}\right)}\|u\|_{F^{s_{2}}}^{\left(r-s_{1}\right) /\left(s_{2}-s_{1}\right)}
$$

which follows immediately from an application of Hölder's inequality.

\section{BLOW UP RATES IN $\dot{H}^{s}, 1 / 2<s<5 / 2, s \neq 3 / 2$}

We now show that the optimal lower bounds in $\dot{H}^{s}$ for $1 / 2<s<5 / 2, s \neq 3 / 2$, can be found directly using the inequalities of Sec. III. First we make the general observation that if

$$
\frac{\mathrm{d}}{\mathrm{d} t} X^{2} \leq \kappa X^{\gamma} \quad \text { for some } \gamma>2,
$$

then

$$
\left[\frac{-1}{[(\gamma / 2)-1]} \frac{1}{X^{\gamma-2}}\right]_{X}^{\infty} \leq t
$$

and so if a solution blows up at time $T$ then

$$
X\left(T^{*}-t\right) \geq \frac{1}{\{[(\gamma / 2)-1] \kappa t\}^{1 /(\gamma-2)}}=c_{\gamma}(\kappa t)^{-1 /(\gamma-2)} .
$$

We make the trivial, but perhaps surprising, remark that an upper bound in a differential inequality leads to a lower bound for any solution that blows up. This explains, in part, the lack of any upper bounds on putative blowup solutions for the Navier-Stokes equations. 
Taking the inner product of equation (3.1) with $u$ in $\dot{H}^{s}$ we obtain, for $1 / 2<s<3 / 2$

$$
\begin{aligned}
\frac{1}{2} \frac{\mathrm{d}}{\mathrm{d} t}\|u\|_{s}^{2}+\|u\|_{s+1}^{2} & \leq c_{s}\|u\|_{s}\|u\|_{s+1}\|u\|_{F} \\
& \leq c_{s}\|u\|_{s}\|u\|_{s+1}\|u\|_{s}^{s-1 / 2}\|u\|_{s+1}^{3 / 2-s} \\
& =c_{s}\|u\|_{s}^{s+1 / 2}\|u\|_{s+1}^{5 / 2-s},
\end{aligned}
$$

where we have used (3.5) and the interpolation (3.10). We obtain the same differential inequality when $3 / 2<s<5 / 2$, since

$$
\begin{aligned}
\frac{1}{2} \frac{\mathrm{d}}{\mathrm{d} t}\|u\|_{s}^{2}+\|u\|_{s+1}^{2} & \leq c_{s}\|u\|_{s}^{2}\|u\|_{F^{1}} \\
& \leq c_{s}\|u\|_{s}^{2}\|u\|_{s}^{s-3 / 2}\|u\|_{s+1}^{5 / 2-s} \\
& =c_{s}\|u\|_{s}^{s+1 / 2}\|u\|_{s+1}^{5 / 2-s},
\end{aligned}
$$

now using (3.6) and (3.10) once more. Application of Young's inequality on the right-hand side yields

$$
\frac{\mathrm{d}}{\mathrm{d} t}\|u\|_{s}^{2} \leq c_{s}\|u\|_{s}^{2(2 s+1) /(2 s-1)}
$$

showing that the local existence time depends only on the norm in $\dot{H}^{s}$ and demonstrating (see (3.7)) that

$$
\|u(T-t)\|_{\dot{H}^{s}} \geq c_{s} t^{-(2 s-1) / 4} .
$$

Thus for $1 / 2<s<3 / 2$ and $3 / 2<s<5 / 2$ we obtain the rate of blowup in (2.2).

\section{THE "BOUNDARY CASES": $s=3 / 2$ AND $s=5 / 2$}

\section{A. Blowup in $\dot{H}^{3 / 2}$ and $\dot{H}^{5 / 2}$}

We cannot quite obtain the optimal blowup rate in $\dot{H}^{3 / 2}\left(\sim t^{-1 / 2}\right)$ and $\dot{H}^{5 / 2}\left(\sim t^{-1}\right)$, but can get arbitrarily close, both on the whole space and in the periodic case.

Taking the inner product of equation (3.1) with $u$ in $\dot{H}^{3 / 2}$ we obtain

$$
\frac{1}{2} \frac{\mathrm{d}}{\mathrm{d} t}\|u\|_{3 / 2}^{2}+\|u\|_{5 / 2}^{2} \leq c\|u\|_{3 / 2}\|u\|_{5 / 2}\|u\|_{F},
$$

using (3.5). If we use the interpolation (3.10) to bound $\|u\|_{F}$ in terms of the norms in $\dot{H}^{3 / 2-\epsilon}$ and $\dot{H}^{3 / 2+\epsilon}$, and then interpolate these homogeneous Sobolev norms, we obtain

$$
\|u\|_{F} \leq c\|u\|_{3 / 2-\epsilon}^{1 / 2}\|u\|_{3 / 2+\epsilon}^{1 / 2} \leq c\|u\|^{\epsilon / 3}\|u\|_{3 / 2}^{1-5 \epsilon / 6}\|u\|_{5 / 2}^{\epsilon / 2} .
$$

Thus

$$
\frac{1}{2} \frac{\mathrm{d}}{\mathrm{d} t}\|u\|_{3 / 2}^{2}+\|u\|_{5 / 2}^{2} \leq c\|u\|^{\epsilon / 3}\|u\|_{3 / 2}^{2-5 \epsilon / 6}\|u\|_{5 / 2}^{1+\epsilon / 2},
$$

which after using Young's inequality yields

$$
\frac{\mathrm{d}}{\mathrm{d} t}\|u\|_{3 / 2}^{2} \leq c\|u\|^{4 \epsilon / 3(2-\epsilon)}\|u\|_{3 / 2}^{4+2 \epsilon / 3(2-\epsilon)}
$$

This is in the form of equation (4.1), so we can immediately deduce that if the solution blows up at time $T^{*}$ then

$$
\left\|u\left(T^{*}-t\right)\right\|_{3 / 2} \geq c_{\epsilon}\left\|u\left(T^{*}-t\right)\right\|_{L^{2}}^{-\epsilon /(3-\epsilon)} t^{-3(2-\epsilon) / 4(3-\epsilon)}
$$

in particular the blowup rate can be made as close to $t^{-1 / 2}$ as required. 
We can use the same technique in $\dot{H}^{5 / 2}$ : if we take the inner product with $u$ in $\dot{H}^{5 / 2}$ and use inequality (3.6) we obtain

$$
\frac{1}{2} \frac{\mathrm{d}}{\mathrm{d} t}\|u\|_{5 / 2}^{2}+\|u\|_{7 / 2}^{2} \leq c\|u\|_{F^{1}}\|u\|_{5 / 2}^{2} .
$$

Then we write

$$
\|u\|_{F^{1}} \leq c\|u\|_{5 / 2-\epsilon}^{1 / 2}\|u\|_{5 / 2+\epsilon}^{1 / 2} \leq c\|u\|^{\epsilon / 5}\|u\|_{5 / 2}^{1-7 \epsilon / 10}\|u\|_{7 / 2}^{\epsilon / 2} .
$$

Thus

$$
\frac{1}{2} \frac{\mathrm{d}}{\mathrm{d} t}\|u\|_{5 / 2}^{2}+\|u\|_{7 / 2}^{2} \leq c\|u\|^{\epsilon / 5}\|u\|_{5 / 2}^{3-7 \epsilon / 10}\|u\|_{7 / 2}^{\epsilon / 2} .
$$

Using Young's inequality we obtain

$$
\frac{\mathrm{d}}{\mathrm{d} t}\|u\|_{5 / 2}^{2} \leq c\|u\|^{4 \epsilon / 5(4-\epsilon)}\|u\|_{5 / 2}^{3+\epsilon / 5(4-\epsilon)} ;
$$

again this in the form of (4.1), and so we can deduce that

$$
\left\|u\left(T^{*}-t\right)\right\|_{5 / 2} \geq c\left\|u\left(T^{*}-t\right)\right\|_{L^{2}}^{-\epsilon /(5-\epsilon)} t^{-5(4-\epsilon) / 4(5-\epsilon)},
$$

with the blowup rate as close to $t^{-1}$ as required.

\section{B. Blowup in $F^{0}$}

If we want to obtain the "optimal rate" in the periodic case in a space that has the same scaling as $\dot{H}^{3 / 2}$, we can follow Benameur (2010) and consider blowup in $F^{0}(Q)$.

Lemma 5.1. There exists an absolute constant $c_{3 / 2}^{\prime}$ such that

$$
\|u(T-t)\|_{F^{0}(Q)} \geq c_{3 / 2}^{\prime} t^{-1 / 2} .
$$

Proof. Taking the Fourier transform of the NSE we have

$$
\frac{\mathrm{d}}{\mathrm{d} t} \hat{u}(k)+|k|^{2} \hat{u}(k)+((u \cdot \nabla) u)^{\wedge}(k)=0 .
$$

Taking the inner product of this equation with $\hat{u}(-k)=\overline{\hat{u}(k)}$ we obtain

$$
\frac{1}{2} \frac{\mathrm{d}}{\mathrm{d} t}|\hat{u}(k)|^{2}+|k|^{2}|\hat{u}(k)|^{2}+((u \cdot \nabla) u)^{\wedge}(k) \cdot \hat{u}(-k)=0 .
$$

Then, dividing by $|\hat{u}(k)|$ (this can be made rigorous by dividing instead by $\sqrt{\left(|\hat{u}(k)|^{2}+\epsilon\right)}$ and letting $\epsilon$ tend to zero) we obtain

$$
\frac{\mathrm{d}}{\mathrm{d} t}|\hat{u}(k)|+|k|^{2}|\hat{u}(k)| \leq\left|((u \cdot \nabla) u)^{\wedge}(k)\right| .
$$

Summing over $k$ gives

$$
\begin{aligned}
\frac{\mathrm{d}}{\mathrm{d} t}\|u\|_{F^{0}}+\|u\|_{F^{2}} & \leq \sum_{k}\left|((u \cdot \nabla) u)^{\wedge}(k)\right| \\
& \leq \sum_{k, l}|\hat{u}(k-l)\|l\| \hat{u}(l)| \\
& \leq\|u\|_{F^{0}}\|u\|_{F^{1}} \\
& \leq\|u\|_{F^{0}}^{3 / 2}\|u\|_{F^{2}}^{1 / 2},
\end{aligned}
$$

using the $F^{r}$ interpolation from (3.11). An application of Young's inequality yields

$$
\frac{\mathrm{d}}{\mathrm{d} t}\|u\|_{F^{0}} \leq\|u\|_{F^{0}}^{3}
$$

and (5.1) follows. 
We note that one can obtain the same result on $\mathbb{R}^{3}$ by considering the solution of (5.2) in integral form,

$$
\hat{u}(k, t)=\mathrm{e}^{-|k|^{2} t} \hat{u}(k, 0)+\int_{0}^{t} \mathrm{e}^{-|k|^{2}(t-s)}((u \cdot \nabla) u)^{\wedge}(k, s) \mathrm{d} s,
$$

whence, since $\hat{u}(l) \cdot l=0$ as $u$ is divergence-free, and using the inequality $\sup _{x>0} x \mathrm{e}^{-t x^{2}} \leq c t^{-1 / 2}$ and the Young inequality for convolutions, we can deduce that

$$
\|\hat{u}(t)\|_{L^{1}} \leq\left\|\hat{u}_{0}\right\|_{L^{1}}+c \int_{0}^{t}(t-s)^{-1 / 2}\|\hat{u}(s)\|_{L^{1}}^{2} \mathrm{~d} s .
$$

Comparing this the solution $X(t)$ of the integral equality

$$
X(t)=X_{0}+c \int_{0}^{t}(t-s)^{-1 / 2} X(s)^{2} \mathrm{~d} s \leq X_{0}+2 c t^{1 / 2} X(t)^{2}
$$

yields an upper bound while $1-8 c X_{0} t^{1 / 2} \geq 0$, i.e. a lower bound on the existence time $T \sim\left\|\hat{u}_{0}\right\|_{L^{1}}^{-2}$, and hence (5.1).

\section{BLOWUP RATES IN $\dot{H}^{s}, s>5 / 2$}

We now consider the case $s>5 / 2$. We gave a proof in Lemma 2.2 of the lower bound

$$
\|u(T-t)\|_{\dot{H}^{s}\left(\mathbb{R}^{3}\right)} \geq c_{s}\|u(T-t)\|_{L^{2}\left(\mathbb{R}^{3}\right)}^{(5-2 s) / 5} t^{-2 s / 5} .
$$

in the whole space, which relied on scaling and the fact that the local existence time is at least $c /\left\|u_{0}\right\|_{H^{s}\left(\mathbb{R}^{3}\right)}$. We first give a simple proof of this lower bound (in terms of the full $H^{s}\left(\mathbb{R}^{3}\right)$ norm), and then a direct proof of (6.1) that is also valid in the periodic case. To obtain the lower bound $c /\left\|u_{0}\right\|_{H^{s}\left(\mathbb{R}^{3}\right)}$, note that for any $k$ it follows from (3.6) that

$$
\frac{1}{2} \frac{\mathrm{d}}{\mathrm{d} t}\|u\|_{k}^{2}+\|u\|_{k+1}^{2} \leq c_{s}\|u\|_{k}^{2}\|u\|_{F^{1}\left(\mathbb{R}^{3}\right)} \leq c_{s}\|u\|_{k}^{2}\|u\|_{H^{s}\left(\mathbb{R}^{3}\right)},
$$

using (3.4). For such an $s$, summing the above inequality for $k=0$ and $k=s$ we obtain

$$
\begin{aligned}
\frac{\mathrm{d}}{\mathrm{d} t}\left(\|u\|_{0}^{2}+\|u\|_{s}^{2}\right) & \leq c_{s}\left(\|u\|_{0}^{2}+\|u\|_{s}^{2}\right)\|u\|_{H^{s}\left(\mathbb{R}^{3}\right)} \\
& \leq c_{s}\left(\|u\|_{0}^{2}+\|u\|_{s}^{2}\right)^{3 / 2}
\end{aligned}
$$

since $\left(\|u\|_{0}^{2}+\|u\|_{s}^{2}\right)^{1 / 2}$ is equivalent to the norm in $H^{s}\left(\mathbb{R}^{3}\right)$. This shows that the local existence time can be bounded below by

$$
c_{s}\left(\|u\|_{0}^{2}+\|u\|_{s}^{2}\right)^{-1 / 2}
$$

and hence by $c_{s}\left\|u_{0}\right\|_{H^{s}}^{-1}$.

Note that for periodic boundary conditions

$$
\frac{1}{2} \frac{\mathrm{d}}{\mathrm{d} t}\|u\|_{s}^{2}+\|u\|_{s+1}^{2} \leq c_{s}\|u\|_{s}^{3}
$$

for any $s>5 / 2$, since in this case we can use (3.3) rather than (3.4), and hence the local existence time in $\dot{H}^{s}(Q)$ depends only on the $\dot{H}^{s}(Q)$ norm. However, the scaling argument used in the whole space case (see Lemma 2.1) relies on being able to choose arbitrarily small scaling factors $\lambda$, and in the periodic case we can only take $\lambda \in \mathbb{N}$ since we are forced to respect the periodic domain $Q$.

We now obtain (6.1) directly using the inequalities from Sec. III, giving an argument that is also valid for periodic boundary conditions. Indeed, if we use (3.10) to interpolate $\|u\|_{F^{1}}$ between $L^{2}$ and $\dot{H}^{s}$ then we obtain

$$
\begin{aligned}
\frac{1}{2} \frac{\mathrm{d}}{\mathrm{d} t}\|u\|_{s}^{2}+\|u\|_{s+1}^{2} & \leq c_{s}\|u\|_{s}^{2}\|u\|_{F^{1}} \\
& \leq c_{s}\|u\|_{s}^{2+(5 / 2 s)}\|u\|_{L^{2}}^{1-(5 / 2 s)}
\end{aligned}
$$


This is in the form of (4.1), so we can deduce the lower bound

$$
\|u(T-t)\|_{s} \geq c_{s}\|u(T-t)\|_{L^{2}}^{(5-2 s) / 5} t^{-2 s / 5} .
$$

Note that if we return to

$$
\frac{1}{2} \frac{\mathrm{d}}{\mathrm{d} t}\|u\|_{k}^{2}+\|u\|_{k+1}^{2} \leq c_{k}\|u\|_{k}^{2}\|D u\|_{F},
$$

(this was (6.2)) then it is clear that the formation of singularities as $t \rightarrow T$ would be prevented by a bound of the form

$$
\int_{0}^{T}\|D u(s)\|_{F} \mathrm{~d} s<\infty .
$$

Since $\|\omega\|_{\infty} \leq\|D u\|_{\infty} \leq\|D u\|_{F}$ this is a weaker form of the well-known Beale-Kato-Majda criterion for regularity,

$$
\int_{0}^{T}\|\omega(s)\|_{L^{\infty}} \mathrm{d} s<\infty
$$

(see Beale et al., 1984).

\section{SOME REMARKS ON UPPER BOUNDS FOR SOLUTIONS}

The "optimal" rate of blowup is closely connected to Leray's conjecture that a putative blowup at a time $T$ could occur for solutions of the form

$$
\tilde{u}(x, t)=\frac{1}{\sqrt{T-t}} U\left(\frac{x}{\sqrt{T-t}}\right) .
$$

Notice that for $\tilde{u}$ given by (7.1) we have

$$
\|\tilde{u}(t)\|_{\dot{H}^{s}\left(\mathbb{R}^{3}\right)}=C(T-t)^{-(2 s-1) / 4},
$$

for all $s>1 / 2$ so the $\dot{H}^{s}\left(\mathbb{R}^{3}\right)$ norm of such self-similar solution blows up exactly at the "optimal" rate. Even though the existence of self-similar solutions given by (7.1) was excluded by Necas et al. (1996), nonetheless one can still consider a solution that blows up as $t$ tends to zero and whose $\dot{H}^{s}\left(\mathbb{R}^{3}\right)$-norm oscillates between $c t^{-(2 s-1) / 4}$ and $C t^{-(2 s-1) / 4}$. So assume that for some solution $u$ and some $\dot{H}^{s}\left(\mathbb{R}^{3}\right)$ we have an upper bound on blow up that matches the lower bound. It turns out that for such solutions we are able to prove the optimal rate of blow up in $\dot{H}^{s}$ not only for $1 / 2<s$ $<5 / 2, s \neq 3 / 2$, but for all $s>1 / 2$.

For example suppose that we have the upper bound

$$
\|u(T-t)\|_{\dot{H}^{1}} \leq C t^{-1 / 4} .
$$

Then simple interpolation gives us

$$
\|u\|_{2} \leq\|u\|_{1}^{(s-2) /(s-1)}\|u\|_{s}^{1 /(s-1)},
$$

so for all $s>2$ we have

$$
\|u(T-t)\|_{s} \geq \frac{\|u(T-t)\|_{2}^{s-1}}{\|u(T-t)\|_{1}^{s-2}} \geq c t^{-3(s-1) / 4} t^{(s-2) / 4}=c t^{-(2 s-1) / 4} .
$$

On the other hand from the inequality

$$
\|u\|_{5 / 4} \leq\|u\|_{1}^{1 / 2}\|u\|_{3 / 2}^{1 / 2}
$$

we can also obtain the "optimal rate" of blow up in $\dot{H}^{3 / 2}$ :

$$
\|u(T-t)\|_{3 / 2} \geq \frac{\|u(T-t)\|_{5 / 4}^{2}}{\|u(T-t)\|_{1}} \geq c t^{-1 / 2} .
$$


Perhaps, even more interestingly an upper bound on the $\dot{H}^{3 / 2}$ norm,

$$
\|u\|_{3 / 2} \leq c t^{-1 / 2},
$$

would yield at most logarithmic growth of the norm in $\dot{H}^{1 / 2}$, since from the bound

$$
\begin{aligned}
\left|\left(B(u, u), A^{1 / 2} u\right)\right| & \leq\|u\|_{L^{6}}\|D u\|_{L^{2}}\left\|A^{1 / 2} u\right\|_{L^{3}} \\
& \leq\|u\|_{1}^{2}\|u\|_{3 / 2} \\
& \leq c\|u\|_{1 / 2}\|u\|_{3 / 2}^{2},
\end{aligned}
$$

we can obtain

$$
\frac{1}{2} \frac{\mathrm{d}}{\mathrm{d} t}\|u\|_{1 / 2}^{2}+\|u\|_{3 / 2}^{2} \leq c\|u\|_{1 / 2}\|u\|_{3 / 2}^{2} \leq c\|u\|_{1 / 2} t^{-1} .
$$

So with $X=\|u\|_{1 / 2}^{2}$ this yields

$$
\frac{\mathrm{d} X}{X^{1 / 2}} \leq c t^{-1}
$$

i.e.,

$$
X(t)^{1 / 2}-X(0)^{1 / 2} \leq c \log \frac{T}{T-t},
$$

so

$$
\|u(T-t)\|_{1 / 2} \leq\left\|u_{0}\right\|_{1 / 2}+c \log \frac{T}{T-t} .
$$

Finally, in connection with possible blowup in $\dot{H}^{1 / 2}$, suppose that we interpolate the $\dot{H}^{1}$ norm between $\dot{H}^{1 / 2}$ and $\dot{H}^{s}$,

$$
\|u\|_{1} \leq\|u\|_{1 / 2}^{(2 s-2) /(2 s-1)}\|u\|_{s}^{1 /(2 s-1)} .
$$

Then

$$
\|u(T-t)\|_{s} \geq \frac{\|u(T-t)\|_{1}^{2 s-1}}{\|u(T-t)\|_{1 / 2}^{2 s-2}} \geq \frac{c}{\|u(T-t)\|_{1 / 2}^{2 s-2}} t^{-(2 s-1) / 4} .
$$

Modulo the factor of the $\dot{H}^{1 / 2}$ in the denominator, this gives the "correct" rate of blowup in $\dot{H}^{s}$. Note, however, that if the solution does blow up as $t \rightarrow T$ then we know Seregin (2011) that the $\dot{H}^{1 / 2}$ norm must blow up, so the rate here is in fact a little slower (perhaps logarithmically?) than $t^{-(2 s-1) / 4}$.

\section{CONCLUSION}

For $1 / 2<s<5 / 2, s \neq 3 / 2$, we have demonstrated that if there is a singularity at time $T$ then solutions must blowup at the "optimal" rate, like $t^{-(2 s-1) / 4}$. For $s=3 / 2$ and $s=5 / 2$ we have only shown that one can get arbitrarily close to the optimal rate. We have also demonstrated, in passing, that for this range of $s(1 / 2<s<5 / 2, s \neq 3 / 2)$ the local existence time for solutions on the whole space can be taken to depend only on the norm in $\dot{H}^{s}\left(\mathbb{R}^{3}\right)$. In a second regime $s>5 / 2$, we have obtained a rate of blowup that is only of the order of $t^{-2 s / 5}$.

A number of interesting open questions immediately present themselves:

(i) Can one prove optimal blowup in $\dot{H}^{3 / 2}$ and $\dot{H}^{5 / 2}$ ?

(i') For $s=3 / 2$ and $s=5 / 2$ can one show that the local existence time in $\dot{H}^{s}\left(\mathbb{R}^{3}\right)$ depends only on the $\dot{H}^{s}$ norm? This would imply (i) on the whole space due to Lemma 2.1.

(ii) Can one find an optimal lower bound in a space with the same scaling as $\dot{H}^{5 / 2}$, e.g., $F^{1}$ ?

(iii) Is the blowup rate in fact optimal for $s>5 / 2$, or is there some mechanism that makes this regime qualitatively different from $s<5 / 2$ ? 
(iv) Can one strengthen inequality (3.6) to

$$
\left|\left(A^{s} B(u, u), u\right)\right| \leq c_{s}\|D u\|_{L^{\infty}}\|u\|_{s}^{2},
$$

i.e., can one replace the $L^{1}$ norm of the Fourier transform of $D u$ in (3.6) by the supremum norm of $D u$ as in (3.9)?

Another very interesting problem is to obtain lower bounds on blowing up solutions on bounded domains, for which the inequalities in Lemma 3.1 are not known to be valid.

\section{ACKNOWLEDGMENTS}

We would all like to thank the anonymous referee for their helpful comments, which led to a number of improvements throughout the paper. This work was carried out while R.P.S. was visiting the Mathematics Institute at Warwick, and he would like to express his gratitude for the hospitality during his time there. J.C.R. is supported by an EPSRC Leadership Fellowship, Grant No. EP/G007470/1; W.S. was supported by grant of the Polish Ministry of Science and Higher Education Grant No. N201 547438; R.P.S. was partially supported by PROPG \UNESP and PROPe \UNESP, Brazil.

Beale, J. T., Kato, T., and Majda, A., "Remarks on the breakdown of smooth solutions for the 3-D Euler equations," Commun. Math. Phys. 94, 61-66 (1984).

Benameur, J., "On the blow-up criterion of 3D Navier-Stokes equations," J. Math. Anal. Appl. 371, 719-727 (2010).

Cannone, M., Ondelettes, Paraproduits et Navier-Stokes (Diderot Editeur, Paris, 1995).

Cannone, M., "Harmonic analysis tools for solving the incompressible Navier-Stokes equations," in Handbook of Mathematical Fluid Dynamics, edited by S. Friedlander and D. Serre (Elsevier, 2003), Vol. 3.

Chemin, J.-Y., Desjardins, B., Gallagher, I., and Grenier, E., Mathematical Geophysics. An Introduction to Rotating Fluids and the Navier-Stokes Equations, Oxford Lecture Series in Mathematics and its Applications Vol. 32 (Oxford University Press, Oxford, 2006).

Constantin, P. and Foias, C., Navier-Stokes Equations (University of Chicago Press, Chicago, 1988).

Fujita, H. and Kato, T., "On the Navier-Stokes initial value problem. I," Arch. Rational Mech. Anal. 16, 269-315 (1964).

Giga, Y., "Solutions for semilinear parabolic equations in $L^{p}$ and regularity of weak solutions of the Navier-Stokes system," J. Differ. Equations 62, 182-212 (1986).

Klainerman, A. and Majda, A., "Singular limits of quasilinear hyperbolic systems with large parameters and the incompressible limit of compressible fluids," Commun. Pure Appl. Math. 34, 481-524 (1981).

Koch, H., and Tataru, D., "Well-posedness for the Navier-Stokes equations," Adv. Math. 157, 22-35 (2001).

Lemarié-Rieusset, P. G., Recent Developments in the Navier-Stokes Problem, Chapman \& Hall/CRC Research Notes in Mathematics Vol. 431 (Chapman \& Hall/CRC, Boca Raton, FL, 2002).

Leray, J., "Sur le mouvement d'un liquide visqueux emplissant l'espace," Acta Math. 63, 193-248 (1934).

Majda, A. J. and Bertozzi, A. L., Vorticity and Incompressible Flow, Cambridge Texts in Applied Mathematics Vol. 27 (Cambridge University Press, Cambridge, 2002).

Marín-Rubio, P., Robinson, J. C., and Sadowski, W., "Solutions of the 3D Navier-Stokes equations for initial data in $H^{1 / 2}$ : robustness of regularity and numerical verification of regularity for bounded sets of initial data in $H^{1}$," J. Math. Anal. Appl., to appear (2012).

Necas, J., Ružicka, M., and Šerák, V., "On Leray’s self-similar solutions of the Navier-Stokes equations,” Acta Math. 176, 283-294 (1996).

Oliver, M. and Titi, E. S., "Remark on the rate of decay of higher order derivatives for solutions to the Navier-Stokes equations in $\mathbb{R}^{n}$," J. Funct. Anal. 172, 1-18 (2000).

Oliver, M. and Titi, E. S., "On the domain of analyticity for solutions of second order analytic nonlinear differential equations," J. Differ. Equations 174, 55-74 (2001).

Robinson, J. C. and Sadowski, W., "A local smoothness criterion for solutions of the 3D Navier-Stokes equations," (submitted).

Seregin, G., "Necessary conditions of potential blowup for the Navier-Stokes equations," J. Math. Sci. 178, 345-352 (2011).

Seregin, G., "A certain necessary condition of potential blow up for Navier-Stokes equations," Comm. Math. Phys. 312, $833-845$ (2012) . 